www.nature.com/pj

\title{
Extension of polyethylene chains by formation of polypseudorotaxane structures with perpentylated pillar[5]arenes
}

\author{
Tomoki Ogoshi, Hitoshi Kayama, Takamichi Aoki, Tada-aki Yamagishi, Ryutaro Ohashi and Motohiro Mizuno \\ Herein, the extension of high-density polyethylene (PE) (HDPE) chains by formation of polypseudorotaxane structures with \\ perpentylated pillar[5]arenes is reported. Melt mixing of polymeric chains of HDPEs and wheels of perpentylated pillar[5]arenes \\ resulted in formation of polypseudorotaxane structures. The formation of the polypseudorotaxane structures led to extension of \\ the HDPE chains, which dramatically increased the melting point of the HDPE from 126 to $152{ }^{\circ} \mathrm{C}$. We also demonstrated \\ molten-to-solid and solid-to-molten state transitions of HDPE based on the host-guest system. HDPE was melted at $140{ }^{\circ} \mathrm{C}$, but \\ changed to a solid upon addition of perpentylated pillar[5]arenes. Further addition of competitive guest 1,4-dibromobutane to \\ the solid mixture induced a solid-to-molten state transition.
}

Polymer Journal (2014) 46, 77-81; doi:10.1038/pj.2013.67; published online 21 August 2013

Keywords: host-guest complex; melting point; pillar[5]arene; polyethylene; polypseudorotaxane

\section{INTRODUCTION}

The crystallinity and morphological features of polyethylene (PE) are important factors affecting its thermal and mechanical properties. ${ }^{1-6}$ Under ambient conditions, PE consists of lamella and amorphous phases. In contrast, when PE crystallizes under special conditions, such as shear, elongation flow and ultrahigh pressure, it forms an extended chain structure. This structure has ultrahigh strength and modulus. Therefore, many studies have attempted to generate the extended chain structure. ${ }^{1,2}$

Pillar[5] arenes (Figure 1), which were first reported by our group, ${ }^{7}$ are cyclic pentamers composed of dialkoxybenzene units connected by methylene bridges at the para positions of the benzene moieties. ${ }^{7-18}$

Pillar[5] arenes have a highly symmetrical cylindrical structure with a $\pi$-electron-rich cavity, and they are ideal hosts for $\pi$-electron poor guests. ${ }^{7-12}$ Interestingly, pillar[5] arenes also bind non-polar linear $n$-alkanes via multiple $\mathrm{CH} / \pi$ interactions in solution and in the solid state. ${ }^{13-15,19,20}$ This interesting discovery led us to extend our study to the formation of polypseudorotaxane structures between the long linear alkyl chain polymer PEs and pillar[5] arenes. Polypseudorotaxanes are constructed via complexation of covalent polymers with macrocycles. Many types of polymers have been employed as axle segments for preparation of polypseudorotaxanes. ${ }^{21,22}$ However, PE-based polypseudorotaxanes have not been reported to date ${ }^{23}$ due to the following two problems with polypseudorotaxane formation between $\mathrm{PE}$ chains and macrocyclic hosts: (1) PE has no polar functional groups to form complexes with hosts and (2) PE has low solubility in organic solvents.

Multiple $\mathrm{CH} / \pi$ interactions between the $\mathrm{C}-\mathrm{H}$ groups of $\mathrm{PE}$ and pillar[5]arenes could be used to overcome (1) and to increase interactions between PE and the hosts. To overcome (2), PE and pillar[5] arene could be complexed via the melt mixing process, which is widely used in industry. $\mathrm{PE}$ is a thermoplastic resin that melts at $126^{\circ} \mathrm{C}$, and it can complex with pillar[5]arene in its molten state. This system, which does not require solvents and $\mathrm{CH} / \pi$ interactions, should be enhanced. ${ }^{16,24-26}$ When various pillar[5] arenes were mixed with molten PE, pillar[5] arenes containing pentyloxy groups ${ }^{17}$ (C5, Figure 1a) highly miscible with PE were formed. This study reports the formation of polypseudorotaxane structures between C5 wheels and PE chains with a high molecular weight. Interestingly, the formation of the polypseudorotaxane structure extended the PE chains and increased the melting point of the PE. Chemically responsive molten state-to-solid and solid-to-molten state transitions of PE based on the host-guest system are also reported.

\section{EXPERIMENTAL PROCEDURE}

Materials

Perpentylated pillar[5]arene (C5) was synthesized according to a previously published protocol. ${ }^{17}$ High-density PE (HDPE, 428019-1KG, melt index $42 \mathrm{~g}$ per $10 \mathrm{~min})$, ultra HDPE $\left(M_{\mathrm{w}}=3000000-6000000,429015-250 \mathrm{G}\right)$, low-density PE (LDPE, $M_{\mathrm{n}}=7700, \quad M_{\mathrm{w}}=35000,427799-250 \mathrm{G}$ ) and polypropylene $\left(M_{\mathrm{n}}=75000, M_{\mathrm{w}}=174000,452149-100 \mathrm{G}\right)$ were purchased from Sigma-Aldrich, St Louis, MO, USA. 
a
Perpentylated Pillar[5]arene (C5)

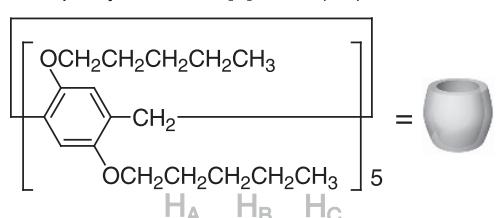

b

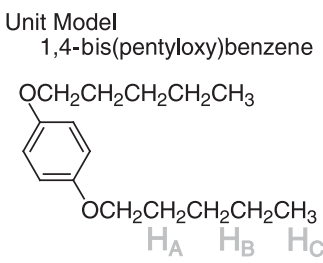

Figure 1 Chemical structures of (a) perpentylated pillar[5]arene (C5) and (b) the unit model 1,4-bis(pentyloxy)benzene. A full color version of this figure is available at Polymer Journal online.

\begin{abstract}
Measurements
${ }^{1} \mathrm{H}$ nuclear magnetic resonance (NMR) spectra were recorded at $500 \mathrm{MHz}$ with a JEOL-ECA500 spectrometer (JEOL, Akishima, Japan). Tapping mode atomic force microscopy (TM-AFM) was performed using a multimode SPA 400 instrument (SEIKO Instruments, Chiba, Japan). Nanoprobe cantilevers (SI-DF20, SEIKO Instruments) were employed. Differential scanning calorimetry (DSC) was performed using a PerkinElmer Jade DSC calorimeter (PerkinElmer, Waltham, MA, USA) equipped with an Intracooler II under a flow of dry nitrogen and with a heating rate of $10^{\circ} \mathrm{C} \mathrm{min}^{-1}$. Thermogravimetric analysis was performed using a TG/DTA6200 thermal analyzer (SEIKO Instruments, Inc.) with a heating rate of $10^{\circ} \mathrm{C} \mathrm{min}{ }^{-1}$ under a nitrogen atmosphere. Small-angle X-ray scattering (SAXS) measurements were performed using the Nanoviewer SAXS system (Rigaku, Akishima, Japan). The finely focused X-ray beam of the $\mathrm{Cu} \mathrm{K} \alpha$ line $(\lambda=1.58 \AA)$ was incident to the sample. Imaging plates were used as detectors for the SAXS data. Wide-angle X-ray scattering (WAXS) measurements were performed using a Smart Lab high-resolution diffractometer (Rigaku). Solid-state NMR experiments were performed at $6.9 \mathrm{~T}\left(74.18 \mathrm{MHz}\right.$ for $\left.{ }^{13} \mathrm{C}\right)$ using a JEOL-ECA-300 NMR spectrometer (JEOL) and a $4.0 \mathrm{~mm}$ CP-MAS probe. All of the samples were spun at a frequency of $5.0 \mathrm{kHz}$. All of the sample temperatures were between 300 and $302 \mathrm{~K}$, calibrated by $\mathrm{Pb}\left(\mathrm{NO}_{3}\right)_{2} \cdot{ }^{18}{ }^{13} \mathrm{C}$ chemical shifts are presented in p.p.m. relative to tetramethylsilane using the ${ }^{13} \mathrm{C}$ chemical shift for the methine ${ }^{13} \mathrm{C}$ of solid adamantine (29.5 p.p.m.) as an external reference standard. For the ${ }^{13} \mathrm{C} 1 \mathrm{D}$ spectra, variable-amplitude cross-polarization ${ }^{27}$ and total elimination of spinning side band ${ }^{28}$ sequences were employed to excite ${ }^{13} \mathrm{C}$ magnetization. In all of the ${ }^{13} \mathrm{C}$ NMR experiments, the ${ }^{13} \mathrm{C}$ signals were detected under ${ }^{1} \mathrm{H}-$ ${ }^{13} \mathrm{C}$ heteronuclear decoupling with the two-pulse phase modulation technique ${ }^{29}$ using a ${ }^{1} \mathrm{H}$ decoupling frequency of $100 \mathrm{kHz}$. In the ${ }^{1} \mathrm{H}-{ }^{13} \mathrm{C}$ heteronuclear two-dimensional experiments, the ${ }^{1} \mathrm{H}$ signals were detected using Phase Modulated Lee-Goldburg homonuclear decoupling. ${ }^{30}$
\end{abstract}

\section{Formation of polypseudorotaxane structures between C5 and HDPE}

Solid samples of $\mathbf{C} 5$ and HDPE were heated to $160^{\circ} \mathrm{C}$, which converted both samples into liquids. These liquids were mixed and stirred at $160^{\circ} \mathrm{C}$ for $10 \mathrm{~min}$. Then, the mixture was cooled to $25^{\circ} \mathrm{C}$.

\section{RESULTS AND DISCUSSION}

Eicosane $n-\mathrm{C}_{20} \mathrm{H}_{42}$ was used as a model compound of HDPE. Because eicosane and $\mathbf{C 5}$ were soluble in chloroform, the complexation was investigated in $\mathrm{CDCl}_{3}$ using ${ }^{1} \mathrm{H}$ NMR (Figure 2).

When eicosane was mixed with $\mathbf{C} 5$ in $\mathrm{CDCl}_{3}$ (Figure $2 \mathrm{~b}$ ), an upfield shift of the peaks for the central $\mathrm{CH}_{2}$ of eicosane (peak $\mathrm{H}_{\mathrm{a}}$ ) was observed, and the proton peak from the terminal $\mathrm{CH}_{3}\left(\right.$ peak $\mathrm{H}_{\mathrm{b}}$ ) varied slightly. In a 2D NOESY study (Supplementary Figure S1), Nuclear Overhauser Effect (NOE) correlations were observed between the central $\mathrm{CH}_{2}$ protons for eicosane (peak $\mathrm{H}_{\mathrm{a}}$ ) and the protons of the pentyloxy and benzene groups of $\mathbf{C 5}$, indicating the formation of the eicosane-C5 complex. The stoichiometry of the eicosane-C5 complex

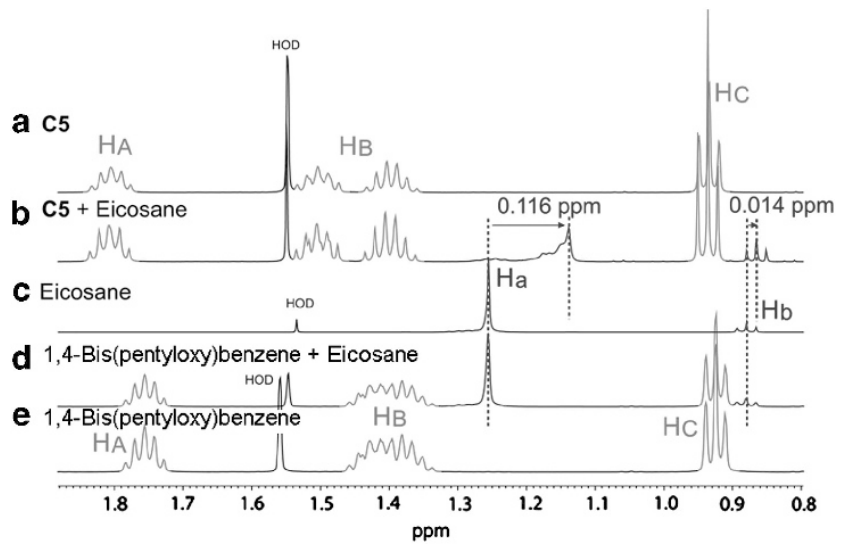

Figure $2{ }^{1} \mathrm{H}$ nuclear magnetic resonance (NMR) spectra of (a) C5 (7 mm), (b) a mixture of $\mathbf{C 5}(7 \mathrm{~mm})$ and eicosane $(3 \mathrm{~mm}),(\mathbf{c})$ eicosane $(3 \mathrm{~mm})$, (d) a mixture of 1,4-bis(pentyloxy)benzene $(35 \mathrm{~mm})$ and eicosane $(3 \mathrm{~mm})$ and (e) 1,4-bis(pentyloxy)benzene $\left(35 \mathrm{~mm}\right.$ ) in $\mathrm{CDCl}_{3}$ at $25^{\circ} \mathrm{C}$. Resonances are labeled as shown in Figure 1. A full color version of this figure is available at Polymer Journal online.

from a Job plot was 1:1 (Supplementary Figure S2). The association constant of the complex, calculated by non-linear fitting of ${ }^{1} \mathrm{H}$ NMR titration of the $\mathrm{CH}_{2}$ protons, was $K=29 \pm 2 \mathrm{M}^{-1}$ (Supplementary Figure S3). In contrast, no peak shifts were observed (Figure $2 \mathrm{~d}$ ) upon mixing eicosane and a unit model of C5, 1,4-bis(pentyloxy)benzene (Figure 1b). This result suggests that there is no interaction between 1,4-bis(pentyloxy)benzene and eicosane. The cyclic structure of pillar[5] arene is needed to form a complex with eicosane via the $\mathrm{CH} / \pi$ interaction and to support complexation of eicosane with C5 in $\mathrm{CDCl}_{3}$.

Next, complexation of eicosane with $\mathbf{C 5}$ in the solid state was investigated. The association constant of the eicosane-C5 complex is relatively weak $\left(K=29 \pm 2 \mathrm{M}^{-1}\right)$, but the complexation might be enhanced in the solid state due to a lack of competitive solvation. ${ }^{16,24-26}$ The complexation was performed via a melt mixing process. When eicosane was heated at $110^{\circ} \mathrm{C}$, the solid eicosane (melting point $36^{\circ} \mathrm{C}$ ) changed to a liquid (Figure 3ai).

C5 was also a liquid at $110^{\circ} \mathrm{C}$ (Figure 3aii) because its melting point is $83^{\circ} \mathrm{C}$. Upon addition of $\mathbf{C 5}$ to liquid eicosane at $110^{\circ} \mathrm{C}$, a transition from the liquid to solid state was observed (Figure 3aiii). The solidification suggests complexation between $\mathbf{C} 5$ and eicosane. The DSC trace of the complex is shown in Figure 3a. The endothermic peak from the melting of eicosane at $36^{\circ} \mathrm{C}$ disappeared, and the endothermic peak from the melting of $\mathbf{C} 5$ at $83^{\circ} \mathrm{C}$ was also barely observed. A new endothermic peak was observed at $135^{\circ} \mathrm{C}$. The endothermic peak of the mixture was higher than those of pristine C5 and eicosane. This result strongly suggests complexation of eicosane with $\mathbf{C 5}$ in the solid state. By contrast, in the sample containing eicosane and 1,4-bis(pentyloxy)benzene, pristine endothermic peaks from eicosane and 1,4-bis(pentyloxy)benzene were observed, but an additional endothermic peak was not observed (Supplementary Figure S4), indicating that there was no interaction between 1,4-bis(pentyloxy)benzene and eicosane. These data are consistent with the complexation of eicosane with $\mathbf{C 5}$ in a solution determined by ${ }^{1} \mathrm{H}$ NMR measurements, and support host-guest complexation between eicosane and $\mathbf{C 5}$ in the solid state.

On the basis of the model complexation system between melted eicosane and C5, the molten-state complexation of HDPE with C5 was investigated. The melting point of HDPE, which has a high 
a
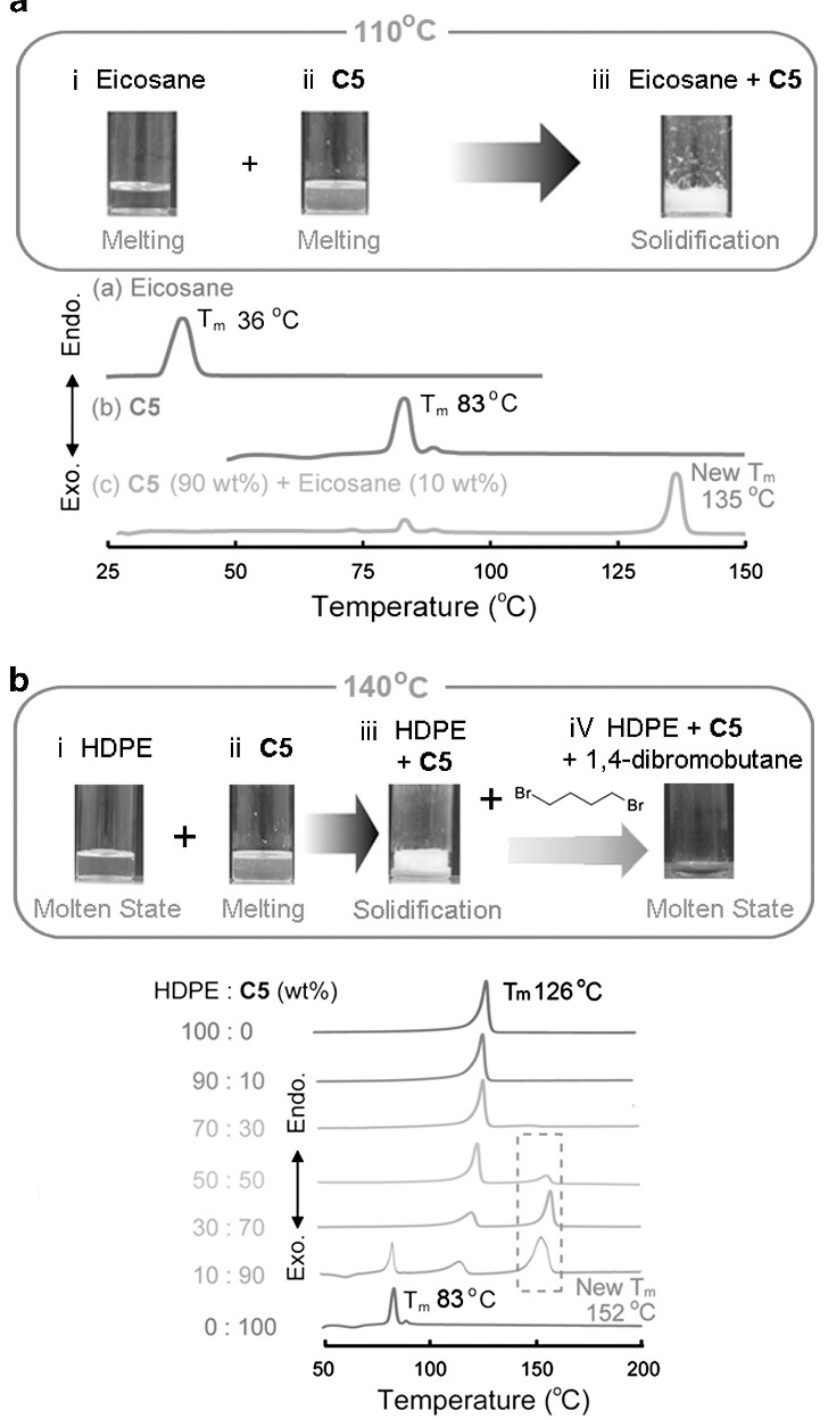

Figure 3 (a) (i-iii) Images at $110^{\circ} \mathrm{C}$ and differential scanning calorimetry (DSC) traces of (i) eicosane, (ii) $\mathbf{C 5}$ and (iii) a mixture of eicosane (10 wt\%) and C5 $(90$ wt\%). (b) Melted state to solid state changes upon mixing of high-density polyethylene (HDPE) with C5 and 1,4-dibromobutane at $140^{\circ} \mathrm{C}$ as well as DSC traces of pristine HDPE, C5 and a mixture of HDPE and C5 with different feed ratios. A full color version of this figure is available at Polymer Journal online.

molecular weight, is $126^{\circ} \mathrm{C}$, and the complexation via melt mixing was performed at $140{ }^{\circ} \mathrm{C}$. Thermogravimetric analysis showed that HDPE and C5 were stable up to $320^{\circ} \mathrm{C}$ (Supplementary Figure S5), and no degradation of these compounds occurred at $140^{\circ} \mathrm{C}$. The molten HDPE at $140{ }^{\circ} \mathrm{C}$ became solid upon addition of $\mathbf{C 5}$ at $140{ }^{\circ} \mathrm{C}$ (Figure $3 \mathrm{~b}$ ), which is the same observation that was made for the mixture of eicosane and C5 (Figure 3a), indicating that complexation has occurred. The complex was investigated using DSC measurements (Figure 3b). A new endothermic peak at $152^{\circ} \mathrm{C}$ appeared along with the pristine endothermic peaks of HDPE and C5 in the samples containing over $50 \mathrm{wt} \% \mathbf{C 5}$. In the sample containing $70 \mathrm{wt} \% \mathbf{C 5}$, the new endothermic peak at $152^{\circ} \mathrm{C}$ was clearly observed with weak endothermic peaks for HDPE melting and no endothermic peak for C5 melting. The sample containing $70 \mathrm{wt} \%$ C5 was the optimum for polypseudorotaxane formation.
The molar percentage of the sample was HDPE:C5 $=97.6$ mol\%:2.4 mol\%. Therefore, $\sim 40 \mathrm{PE}$ units were covered by a C5 unit. The small amount of $\mathbf{C 5}$ is enough to expand the PE chains. These DSC traces did not change with multicycle scanning (Supplementary Figure S6) and were independent of the heating rate (Supplementary Figure S7). This result indicates that the DSC traces are completely reproducible, and that the heating conditions do not affect the melting points. Therefore, the new endothermic peak could be attributed to the host-guest complex between $\mathbf{C 5}$ and HDPE. The data support the formation of polypseudorotaxanes of HDPE with C5 in the solid state. The observation of the cross-peak between HDPE and $\mathbf{C} 5$ by Phase Modulated Lee-Goldburg $\left({ }^{1} \mathrm{H}-{ }^{13} \mathrm{C}\right)$ 2D HETCOR studies also supports complexation of HDPE with $\mathbf{C} 5$ in the solid state (Supplementary Figure S8).

Polypropylene, which has a branched structure, was employed as a guest polymer. In the DSC measurements (Supplementary Figure S9), the endothermic peaks from pristine $\mathbf{C 5}$ and polypropylene were observed at 83 and $122^{\circ} \mathrm{C}$, respectively, but no new endothermic peaks were observed at higher temperatures. This result indicates that complexation did not occur between polypropylene and C5. In a previous study, pillar[5] arene was found to form complexes with linear alkanes but not with cyclic and branched alkanes. ${ }^{13}$ Therefore, complexation between branched polypropylene and C5 may not occur. C5 selectively formed polypseudorotaxanes with linear HDPE in the bulk state and increased the melting point of HDPE. When LDPE was used as a guest polymer, a new endothermic peak was observed at $130-140^{\circ} \mathrm{C}$ in mixtures of LDPE and C5 (Supplementary Figure S10). However, in comparison with a mixture of HDPE and C5, the new additional peak was broadened, and the pristine endothermic peak of LDPE was also observed at $92{ }^{\circ} \mathrm{C}$. The polypseudorotaxane structures were partially formed even though un-complexed LDPE chains remained due to the branched structures of LDPE. In addition, we verified the molecular weight effect. When ultra HDPE $\left(M_{\mathrm{w}}=3000000-6000000\right)$ was employed, the same new endothermic peak at $152{ }^{\circ} \mathrm{C}$ was observed (Supplementary Figure $\mathrm{S} 11)$. Therefore, $\mathrm{PE}$ formed a polypseudorotaxane structure in the low molecular weight oligomeric linear alkane (eicosane) as well as in HDPE with an ultrahigh molecular weight.

In previous studies, the formation of extended PE chains increased the melting point and led to observation of dual melting points of HDPE. ${ }^{4-6}$ We also found dual melting endotherms at 126 and $152^{\circ} \mathrm{C}$ in a mixture of HDPE and C5. Therefore, the formation of polypseudorotaxane structures by mixing HDPE and C5 should expand the folded lamellae to an extended linear chain structure. To investigate the polypseudorotaxane structure constructed from C5 and HDPE, the morphology and periodic structure were measured using WAXS, SAXS and TM-AFM (Figure 4).

C5 exhibited spherical architectures in the TM-AFM measurements (Figure $4 \mathrm{~d}$ ). Two diffraction peaks at $q=0.38(1.7 \mathrm{~nm})$ and 0.42 $(1.45 \mathrm{~nm})$ were observed by SAXS. The diffraction peaks at 1.7 and $1.45 \mathrm{~nm}$ correspond to the longitudinal and lateral periodic structures, respectively, of $\mathbf{C 5}$, which coincides with the X-ray crystal structure of C5. ${ }^{17}$ In HDPE (Figure 4a), the TM-AFM image showed an individual lamellae phase. In the height profile (Figure 4a, insert), the peak-to-valley distance was $15-20 \mathrm{~nm}$. The thickness of the lamellae determined by SAXS was $22.6 \mathrm{~nm}$, which agrees with the thickness of the lamellae determined by the height profile. These data indicate that HDPE consists of lamellae and amorphous phases (Figure 5a).

In the WAXS measurement, reflection peaks were observed at $2 \theta=21.5$ and 23.9. These peaks were assigned as typical 


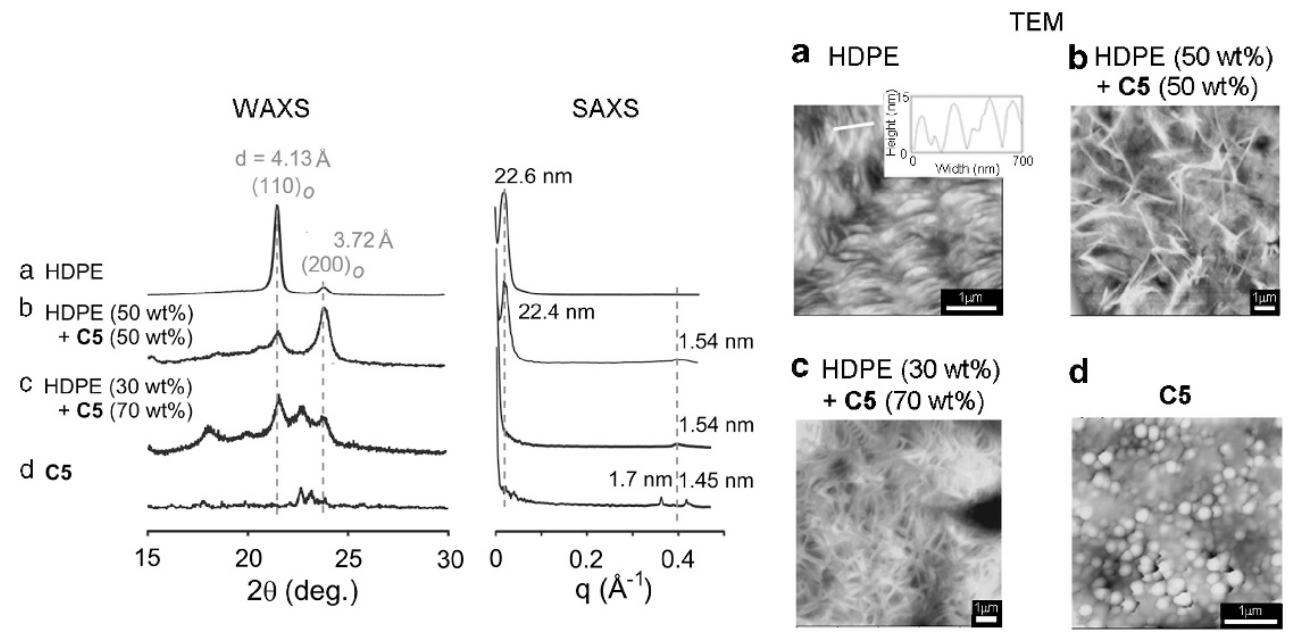

Figure 4 Tapping mode atomic force microscopy (TM-AFM), small-angle X-ray scattering (SAXS) and wide-angle X-ray scattering (WAXS) studies of (a) highdensity polyethylene (HDPE), (b) a mixture of C5 (50 wt\%) and HDPE (50 wt\%), (c) a mixture of C5 (70 wt \%) and HDPE (30 wt\%), and (d) C5. A full color version of this figure is available at Polymer Journal online.

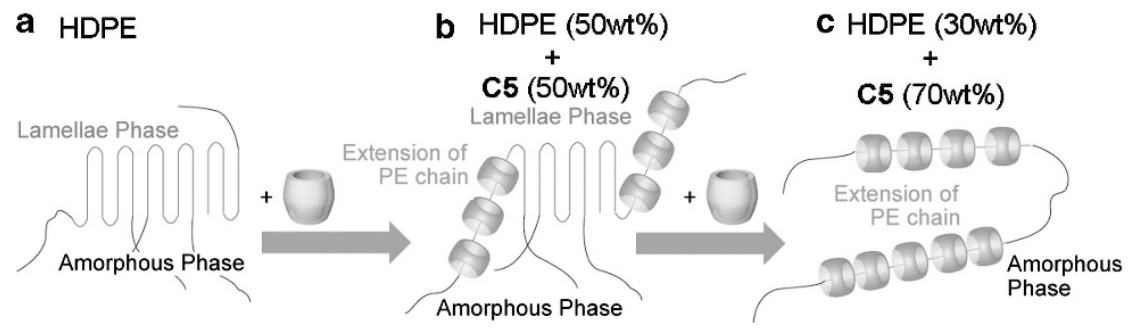

Figure 5 Proposed structure of the high-density polyethylene (HDPE) (a) without $\mathbf{C 5}$ and $(\mathbf{b}, \mathbf{c})$ with $\mathbf{C 5}$. A full color version of this figure is available at Polymer Journal online.

orthorhombic $(110)_{\mathrm{o}}$ and $(200)_{\mathrm{o}}$ reflections. ${ }^{5,6}$ In the sample containing HDPE and $50 \mathrm{wt} \%$ C5 (Figure 4b), extended wires were detected in the lamellar phase matrix by TM-AFM. Using the SAXS measurement, a new diffraction peak $(1.54 \mathrm{~nm})$ was detected along with the peak from the lamellar phase $(22.4 \mathrm{~nm})$. The new peak was assigned to the diffraction of the polypseudorotaxanes, which were composed of wire assemblies. Therefore, the sample is a mixture of lamellae and amorphous phases and has an extended structure of PE chains (Figure 5b). In the sample containing HDPE and $70 \mathrm{wt} \% \mathbf{C 5}$ (Figure 4c), wire-shaped assemblies were clearly observed by TMAFM. In the SAXS measurements, the peak from the lamellae phase $(22.4 \mathrm{~nm})$ completely disappeared, and a diffraction peak at $1.55 \mathrm{~nm}$ was detected. In the WAXS measurement, the intensity of the orthorhombic $(110)_{\mathrm{o}}$ and $(200)_{\mathrm{o}}$ reflections decreased, indicating a decrease in the typical orthorhombic phase of HDPE upon addition of C5. These data indicate the disappearance of the lamellae phase and expansion of the PE chains (Figure $5 \mathrm{c}$ ).

1,4-Dibromobutane forms stable 1:1 host-guest complexes with pillar[5] arenes $\left(K=1.6-4.9 \times 10^{3} \mathrm{M}^{-1}\right)$, which has a larger association constant than the eicosane-C5 complex $\left(K=29 \pm 2 \mathrm{M}^{-1}\right) .^{31}$ When a competitive guest (that is, 1,4-dibromobutane) was added to the solid mixture of HDPE and $\mathbf{C 5}$ at $140{ }^{\circ} \mathrm{C}$, the solid changed to a molten state (Figure 3biv). The transition was monitored by DSC measurements (Supplementary Figure S12). Upon addition of 1,4dibromobutane, the new endothermic peak at $152^{\circ} \mathrm{C}$ due to the formation of the polypseudorotaxane decreased, and the pristine endothermic peak from HDPE reappeared. This result indicates that the polypseudorotaxane constructed from HDPE and C5 dissociated because 1,4-dibromobutane more strongly interacts with $\mathbf{C 5}$ than $n$-with alkanes. On the basis of the host-guest complexation system, solid-to-molten state transitions can be achieved. The transitions induced upon addition of the competitive guest also support the complexation of HDPE and C5 in the solid state.

\section{CONCLUSIONS}

A polypseudorotaxane structure was formed by the mixing of $\mathbf{C 5}$ with HDPE in the melted state. To the best of our knowledge, this is the first example of the formation of polypseudorotaxane structures based on PE. The pseudopolyrotaxanation led to the extension of the PE chains, which dramatically increased the melting point of HDPE from 126 to $152{ }^{\circ} \mathrm{C}$. To date, physical forces, such as shear, elongation flow and ultrahigh pressure, have been necessary to extend PE chains. ${ }^{5,6} \mathrm{~A}$ new chemical method was developed in this study for the extension of $\mathrm{PE}$ chains via the melt mixing process. This process is a new method for host-guest complexation that is very simple and industrially useful for enhancing the thermal properties of HDPE. Furthermore, this study demonstrated that chemically responsive molten state-to-solid and solid-to-molten state transitions were possible by alternating the addition of $\mathbf{C 5}$ and the competitive guest 1,4-dibromobutane. These phase transitions could be applied to new molding and processing techniques of HDPE. 


\section{ACKNOWLEDGEMENTS}

This work was partly supported by The Japan Securities Scholarship Foundation and Technology (MEXT), Japan and JSPS KAKENHI 23655210 (a Grant-in-Aid for Challenging Exploratory Research).

1 Yang, Y., Priyadarshani, N., Khamatnurova, T., Suriboot, J. \& Bergbreiter, D. E. Polyethylene as a nonvolatile solid cosolvent phase for catalyst separation and recovery. J. Am. Chem. Soc. 134, 14714-14717 (2012).

2 Cheng, S., Chen, X., Hsuan, Y. G. \& Li, C. Y. Reduced graphene oxide-induced polyethylene crystallization in solution and nanocomposites. Macromolecules 45 , 993-1000 (2012)

3 Nakae, M., Uehara, H., Kanamoto, T., Zachariades, A. E. \& Porter, R. S. Structure development upon melt drawing of ultrahigh molecular weight polyethylene: effect of prior thermal history. Macromolecules 33, 2632-2641 (2000).

4 Uehara, H., Kanamoto, T., Kawaguchi, A. \& Murakami, S. Real-time X-ray diffraction study on two-stage drawing of ultra-high molecular weight polyethylene reactor powder above the static melting temperature. Macromolecules 29, 1540-1547 (1996).

5 Mandelkern, L. Crystallization of Polymers (Cambridge University Press, Cambridge, UK, 2002).

6 Schulz, J. M. Polymer Crystallization (Oxford University Press, New York, 2001).

7 Ogoshi, T., Kanai, S., Fujinami, S., Yamagishi, T. \& Nakamoto, Y. Para-bridged symmetrical pillar[5]arenes: their Lewis acid catalyzed synthesis and host-guest property. J. Am. Chem. Soc. 130, 5022-5023 (2008).

8 Ogoshi, T. Synthesis of novel pillar-shaped cavitands 'pillar[5]arenes' and their application for supramolecular materials. J. Incl. Phenom. Macrocycl. Chem. 72, 247-262 (2012).

9 Ogoshi, T. \& Yamagishi, T. New synthetic host pillararenes: their synthesis and application to supramolecular materials. Bull. Chem. Soc. Jpn. 86, 312-332 (2013).

10 Ogoshi, T. \& Yamagishi, T. Pillararenes: versatile synthetic receptors for supramolecular chemistry. Eur. J. Org. Chem. 15, 2961-2975 (2013).

11 Cragg, P. J. \& Sharma, K. Pillar[5]arenes: fascinating cyclophanes with a bright future. Chem. Soc. Rev. 41, 597-607 (2012).

12 Xue, M., Yang, Y., Chi, X., Zhang, Z. \& Huang, F. Pillararenes, a new class of macrocycles for supramolecular chemistry. Acc. Chem. Res, 45, 1294-1308 (2012).

13 Ogoshi, T., Demachi, K., Kitajima, K. \& Yamagishi, T. Selective complexation of n-Alkanes with pillar[5]arene dimers in organic media. Chem. Commun. 47, 10290-10292 (2011).

14 Zhang, Z., Luo, Y., Chen, J., Dong, S., Yu, Y., Ma, Z. \& Huang, F. Formation of linear supramolecular polymers that is driven by $\mathrm{C}-\mathrm{H} \cdots \pi$ interactions in solution and in the solid state. Angew. Chem. Int. Ed. 50, 1397-1401 (2011).
15 Strutt, N. L., Forgan, R. S., Spruell, J. M., Botros, Y. Y. \& Stoddart, J. F. Monofunctionalized pillar[5]arene as a host for alkanediamines. J. Am. Chem. Soc. 133, 5668-5671 (2011).

16 Ogoshi, T., Aoki, T., Shiga, R., lizuka, R., Ueda, S., Demachi, K., Yamafuji, D., Kayama, H. \& Yamagishi, T. Cyclic host liquids for facile and high-yield synthesis of [2]rotaxanes. J. Am. Chem. Soc. 134, 20322-20325 (2012).

17 Ogoshi, T., Kitajima, K., Aoki, T., Fujinami, S., Yamagishi, T. \& Nakamoto, T. Synthesis and conformational characteristics of alkyl-substituted pillar[5]arenes. J. Org. Chem. 75, 3268-3273 (2010).

18 Takahashi, T., Kawashima, H., Sugisawa, H. \& Baba, T. 207Pb chemical shift thermometer at high temperature for magic angle spinning experiments. Solid State NMR 15, 119-123 (1999).

19 Takahashi, O., Kohno, Y. \& Nishio, M. Relevance of weak hydrogen bonds in the conformation of organic compounds and bioconjugates: evidence from recent experimental data and high-level ab initio MO calculations. Chem. Rev. 110, 6049-6076 (2010).

20 Nishio, M. CH/ $\pi$ hydrogen bonds in crystals. Cryst. Eng. Commun. 6, 130-158 (2004).

21 Harada, A., Hashidzume, A., Yamaguchi, H. \& Takashima, Y. Polymeric rotaxanes. Chem. Rev. 109, 5974-6023 (2009).

22 Wenz, G., Han, B. H. \& Müller, A. Cyclodextrin rotaxanes and polyrotaxanes. Chem. Rev. 106, 782-817 (2006).

$23 \mathrm{Li}$, J., Harada, A. \& Kamachi, M. Formation of inclusion complexes of oligoethylene and its derivatives with $\alpha$-cyclodextrin. Bull. Chem. Soc. Jpn 67, 2808-2818 (1994).

24 Sozzani, P. Comotti, A. Bracco, S. \& Simonutti, R. Cooperation of multiple $\mathrm{CH} / \pi$ interactions to stabilize polymers in aromatic nanochannels as indicated by $2 \mathrm{D}$ solid state NMR. Chem. Commun. 768-769 (2004).

25 Comotti, A., Simonutti, R., Catel, G. \& Sozzani, P. Isolated linear alkanes in aromatic nanochannels. Chem. Mater. 11, 1476-1483 (1999).

26 Sozzani, P., Comotti, A., Bracco, S. \& Simonutti, R. A family of supramolecular frameworks of polyconjugated molecules hosted in aromatic nanochannels. Angew. Chem. Int. Ed. 43, 2792-2797 (2004).

27 Peersen, O. B., Wu, X., Kustanovich, I. \& Smith, S. O. Variable-amplitude crosspolarization MAS NMR. J. Magn. Reson. A 104, 334-339 (1993).

28 Dixon, W. T., Schaefer, J. S., Sefcik, M. D., Stejskal, E. O. \& McKay, R. A. Tota suppression of sidebands in CPMAS C-13 NMR. J. Magn. Reson. 49, 341-345 (1982).

29 Bennett, A. E., Rienstra, C. M., Auger, M., Lakshmi, K. V. \& Griffin, R. G. Heteronuclear decoupling in rotating solids. J. Chem. Phys. 103, 6951-6958 (1995).

30 Vinogradov, E., Madhu, P. K. \& Vega, S. High-resolution proton solid-state NMR spectroscopy by phase-modulated Lee-Goldburg experiment. Chem. Phys. Lett. 314, 443-450 (1999).

31 Shu, X., Fan, J., Li, J., Wang, X., Chen, W., Jia, X. \& Li, C. Complexation of neutral 1, 4-dihalobutanes with simple pillar[5]arenes that is dominated by dispersion forces. Org. Biomol. Chem. 10, 3393-3397 (2012)

Supplementary Information accompanies the paper on Polymer Journal website (http://www.nature.com/pj) 